\title{
Interval timing deficits and abnormal cognitive development
}

\author{
Christine M. Falter ${ }^{1 *}$ and Valdas Noreika ${ }^{1,2}$ \\ Department of Psychiatry, University of Oxford, Oxford, UK \\ 2 Department of Psychology, Centre for Cognitive Neuroscience, University of Turku, Turku, Finland \\ *Correspondence: christine.falter@psych.ox.ac.uk
}

Time perception deficits represent an aspect of cognitive malfunctioning shared by developmental disorders, which otherwise seem distinct with respect to their individual primary symptom clusters, such as autism spectrum disorders (ASD) and attention-deficit/hyperactivity disorder (ADHD). Multiple impairments of social interaction, communication, and restricted interests constitute the diagnostic criteria for ASD, whereas behavioral symptoms of ADHD comprise inattention, hyperactivity, and impulsivity (DSM-IV-TR; American Psychiatric Association, 2000). Both ASD and ADHD are additionally associated with non-diagnostic secondary symptoms in perception and cognition. A secondary symptom observed in both ASD and ADHD is abnormal interval timing, i.e., processing of stimulus duration (for a review see Falter and Noreika, accepted). For instance, it has been reported that reproduction of auditory and visual intervals of 1-5.5 s was impaired in individuals with ASD (Szelag et al., 2004). Similarly, Barkley et al. (2001) found that individuals with ADHD were impaired in reproducing intervals of 2-60 s.

It is difficult to assess whether secondary symptoms in general and interval timing abnormalities in particular play a causal role in developmental disorders. There are several possible relationships. First, a direct causal relationship would characterize a case in which impairment of an interval timing system could directly disrupt (otherwise possibly unimpaired) cognitive functions relying on accurate timing information, resulting in the known primary symptoms. Although such a direct causal relationship might be found in adult neuropsychology, it is not applicable to the study of developmental disorders, which are characterized by atypical neurogenetic pathways of cognitive development. Rather, as a second option, an ontogenetic causal relationship is conceivable in which an impaired interval timing system could affect the development of all processes downstream, which rely on accurate timing information. Finally, a third option would be that abnormal interval timing could be an epiphenomenon of developmental disorders without bearing a causal relationship to other symptoms.

The idea of an ontogenetic causal relationship raises the question of why the phenomenological outcomes of the disorders differ so strongly. The apparent lack of specificity of interval timing deficits to ASD and ADHD raises the question of their explanatory relevance for the ontogenesis of a particular developmental disorder. A further challenge for the causality assumption is the lack of universality of interval timing abnormalities. A few studies report null findings (Wallace and Happe, 2008; Jones et al., 2009), and in studies showing group differences not all individuals with ASD or ADHD perform atypically. Indeed, the range of reproduced time intervals can be quite variable and the variability is often increased in ASD and ADHD compared to typically developing controls (Toplak et al., 2003; Martin et al., 2010). At the current stage of knowledge, therefore, it seems premature to suggest that interval timing abnormalities can be the sole ontogenetic cause of ASD or ADHD.

Nevertheless, before we discard any causal account in favor of the alternative view of interval timing as a mere epiphenomenon of atypical cognitive development, we suggest that a more associative view of the role of interval timing in cognitive development be adopted. Indeed, there is strong evidence for the association between temporal processing and other typical cognitive functions such as social cognition (Trevarthen and Daniel, 2005; Striano et al., 2006), language processing (Tallal et al., 1993), and understanding of causality (Freeman, 2008). Therefore, it is difficult to conceive how an impairment of interval timing would have no relevance for developmental disorders, which show deficits in cognitive functions relying on accurate timing. Furthermore, the incidence of interval timing abnormalities is increased in developmental disorders, as shown by group differences in perfor- mance even for relatively small sample sizes (Szelag et al., 2004). Thus, in spite of the lack of universality of interval timing deficits, the increased incidence rate needs to be explained. Moreover, it has been suggested that ADHD and ASD share some susceptibility genes (Castellanos and Tannock, 2002), which makes it likely that some dysfunctions are shared between them or their subtypes. We propose that the focus of research needs to be on the association of interval timing abnormalities and other functional deficits. For instance, although a Theory of Mind deficit can hamper the understanding of social situations in its own right, an additional interval timing deficit could result in a lack of precise perception of temporal cues of eye gaze, and thereby increase misinterpretations of social situations. It has been proposed that different symptoms can be independent dimensions of impairment, which nevertheless interact with and modulate one another, leading to the characteristic phenomenology of an individual with a developmental disorder (Happe et al., 2006). In this line of thought, interval timing abnormalities might interact with primary dysfunctions.

In fact, several ADHD studies confirmed significant associations between duration perception and other cognitive functions. Toplak and Tannock (2005) reported significant correlations between time discrimination thresholds and working memory measures in participants with ADHD, but not in healthy individuals. Rubia and colleagues argued persuasively that the primary ADHD symptom of impulsiveness is based on poor inhibition and attention functions, as well as on poor interval timing (Rubia, 2002; Rubia et al., 2009). Even though interaction between interval timing and other cognitive functions is much less investigated in ASD than in ADHD, preliminary findings show significant correlation between timing measures in a temporal bisection task and primary ASD symptoms in the language and communication domain (Allman et al., 2011). 
Taken together, these reports demonstrate complex associations between abnormally developing cognitive functions, and suggest that interval timing might play an important yet under-investigated role in developmental disorders by interacting with and modulating primary symptoms.

\section{ACKNOWLEDGMENTS}

Christine M. Falter is supported by the German Research Council and the Baily Thomas Charitable Fund. Valdas Noreika is supported by the Academy of Finland, the Signe and Ane Gyllenberg Foundation, and the National Graduate School of Psychology in Finland. The collaboration between Christine M. Falter and Valdas Noreika is supported by a European Cooperation in Science and Technology (COST) action on Time in Mental Activity (TIMELY; TD0904).

\section{REFERENCES}

Allman, M., DeLeon, I. G., and Wearden, J. H. (2011). A psychophysical assessment of timing in individuals with autism. Am. J. Intellect. Dev. Disabil. 116, 165-178.

American Psychiatric Association. (2000). Diagnostic and Statistical Manual of Mental Disorders, 4 th Edn. - Test Revision (DSM-IV-TR). Washington, DC: American Psychiatric Association
Barkley, R. A., Murphy, K. R., and Bush, T. (2001). Time processing and reproduction in young adults with attention deficit hyperactivity disorder. Neuropsychology 15, 351-360.

Castellanos, F.X., and Tannock, R. (2002). Neuroscience of attention-deficit/hyperactivity disorder: the search for endophenotypes. Nat. Rev. Neurosci. 3 , 617-628.

Freeman, W. J. (2008). Perception of time and causation through the kinesthesia of intentional action. Integr. Psychol. Behav. Sci. 42, 137-143.

Happe, F., Ronald, A., and Plomin, R. (2006). Time to give up on a single explanation for autism. Nat. Neurosci. 9, 1218-1220.

Jones, C. R. G., Happé, F., Baird, G., Simonoff, E., Marsden, A. J. S., Tregay, J., Phillips, R. J., Goswami, U., Thomson, J.M., and Charman, T. (2009). Auditory discrimination and auditory sensory behaviours in autism spectrum disorders. Neuropsychologia 47 , 2850-2858.

Martin, J. S., Poirier, M., and Bowler, D. M. (2010). Brief report: impaired temporal reproduction performance in adults with autism spectrum disorder. J. Autism Dev. Disord. 40, 640-646.

Rubia, K. (2002). The dynamic approach to neurodevelopmental psychiatric disorders: Use of fMRI combined with neuropsychology to elucidate the dynamics of psychiatric disorders, exemplified in ADHD and schizophrenia. Behav. Brain Res. 130, $47-56$.

Rubia, K., Halari, R., Christakou,A., and Taylor,E. (2009). Impulsiveness as a timing disturbance: neurocognitive abnormalities in attention-deficit hyperactivity disorder during temporal processes and normalization with methylphenidate. Philos. Trans. R. Soc. Lond. B Biol. Sci. 364, 1919-1931.
Striano, T., Henning, A., and Stahl, D. (2006). Sensitivity to interpersonal timing at 3 and 6 months of age. Interact. Stud. 7, 251-271.

Szelag, E., Kowalska, J., Galkowski, T., and Pöppel, E. (2004). Temporal processing deficits in high-functioning children with autism. Br. J. Psychol. 95, 269-282.

Tallal,P., Miller, S., and Fitch, R. H. (1993). Neurobiological basis of speech: a case for the preeminence of temporal processing. Ann. N. Y. Acad. Sci. 14, 27-47.

Toplak, M. E., Rucklidge, J. J., Hetherington, R., John, S. C. F., and Tannock, R. (2003). Time perception deficits in attention-deficit/hyperactivity disorder and comorbid reading difficulties in child and adolescent samples. J. Child Psychol. Psychiatry 44, 888-903.

Toplak, M. E., and Tannock, R. (2005). Time perception: modality and duration effects in attention-deficit/ hyperactivity disorder (ADHD). J. Abnorm. Child. Psychol. 33, 639-654.

Trevarthen, C., and Daniel, S. (2005). Disorganized rhythm and synchrony: early signs of autism and Rett syndrome. Brain Dev. 27(Suppl. 1), 25-34.

Wallace, G. L., and Happe, F. (2008). Time perception in autism spectrum disorders. Res. Autism Spectr. Disord. 2, 447-455.

Received: 31 May 2011; accepted: 03 June 2011; published online: 13 June 2011.

Citation: Falter CM and Noreika V (2011) Interval timing deficits and abnormal cognitive development. Front. Integr. Neurosci. 5:26. doi: 10.3389/fnint.2011.00026

Copyright $\odot 2011$ Falter and Noreika. This is an open-access article subject to a non-exclusive license between the authors and Frontiers Media SA, which permits use, distribution and reproduction in other forums, provided the original authors and source are credited and other Frontiers conditions are complied with. 